\title{
ETAT DU MAGNESIUM DANS QUELQUES SOLS SALES DU SUD ET DU CENTRE DE L'IRAK
}

\author{
A. LARIBI' et A. H. ALZUBAIDI ${ }^{2}$ \\ ${ }^{1}$ BP 68 BIS 16320 DELY IBRAHIM-ALGER. ALGERIA. \\ ${ }^{2}$ COLLEGEOFAGRICULTURE-ABU GHRIB-BAGHDAD. IRAK.
}

\begin{abstract}
RESUME
Le présent travail évalue l'état du magnésium $(\mathrm{Mg})$ dans quelques sols salés. L'étude a été réalisée sur 86 échantillons de sols provenant du Centre et du Sud de l'Irak. L'expérimentation a consisté, d'une part, à calculer les différents sels dominants dans les sols salés par la méthode de combinaison hypothétique et, d'autre part, à déterminer des différentes espèces ioniques et des minéraux contrôlant l'activité de Mg. Pour ce dernier point, un logiciel informatique Visual Minteq, dont le principe est basé sur un concept thermodynamique a été utilisé. Les résultats montrent des teneurs en $\mathrm{Mg}$ supérieures aux limites de tolérance de la plupart des des cultures. La teneur en $\mathrm{Mg}$ soluble, par rapport à la somme des cations dans la solution du sol, a varié entre 12,84 et $34,63 \%$, soit en moyenne $26,2 \%$. Les combinaisons hypothétiques, ont révélé que la majorité des sels de $\mathrm{Mg}$ sont sous forme de $\mathrm{MgCl}_{2}, \mathrm{MgSO}_{4}$, avec une quantité négligeable sous forme de $\mathrm{MgHCO}_{3}$. La teneur moyenne du mélange $\left(\mathrm{MgSO}_{4}+\mathrm{MgCl}_{2}+\mathrm{Mg}\left(\mathrm{HCO}_{3}\right)_{2}\right)$ a été de l'ordre de $25,6 \%$ et a représenté la valeur moyenne des sels de $\mathrm{Mg}$ dominant dans les sols salés. Le logiciel a permis de révélé la présence des espèces ioniques suivantes : $\mathrm{Mg}^{+2}, \mathrm{MgSO}_{4}^{0}, \mathrm{MgCl}^{+}, \mathrm{Mg}_{2} \mathrm{CO}_{3}^{+2}$ et $\mathrm{MgCO}_{3}^{0}$. Les teneurs moyennes de ces espèces, par rapport à $\mathrm{Mg}$ solubletotal ont été de : 40,91\%, 20,69\%, 38,22 \%, $0,064 \%$ et $0,105 \%$, respectivement. Enfin, le programme a montré que les minéraux qui contrôlent l'activité de $\mathrm{Mg}$ dans les sols salés sont, soit des calcaires riches en Mg (Mg-bearing calcite), soit de la dolomite.
\end{abstract}

Mots clés : Statut, magnésium, sels, formes ioniques, minéraux,Irak.

\author{
ABSTRACT \\ STATUS OF MAGNESIUM IN SOME SALINE SOILS FROM THE MIDDLE AND SOUTHERN \\ PARTS OF IRAQ
}

This work, aiming at evaluating the status of magnesium $(\mathrm{Mg})$ in saline soils from the middle and southern parts of Iraq. Dominant salts were assessed using a Visual Minteq data-processing software and the the ionic species and types of minerals controlling $\mathrm{Mg}$ activity calculated. Result show that $\mathrm{Mg}$ levels in saline soils were relatively high, with values exceeding the critical limits for most crops. Total Mg content over total cations in soil solution ranged between $12,84-34,63 \%$, with an average $26,2 \%$. The hypothetical combination, data showed that the majority of $\mathrm{Mg}$ salts were in the form of $\mathrm{MgCl}_{2}$ and $\mathrm{MgSO}_{4}$ salts, with negligible quantities of $\mathrm{MgHCO}_{3}$. The average proportion of the mixture $\left(\mathrm{MgSO}_{4}+\mathrm{MgCl}_{2}+\mathrm{MgHCO}_{3}\right)$ was about 25,6\%, which represented the average value of the dominating $\mathrm{Mg}$ salts in saline soils. The use of the Visual Minteq program allowed to identify the presence of the following $\mathrm{Mg}$ species in the soil solution: $\mathrm{Mg}^{+2}, \mathrm{MgSO}_{4}{ }^{0}, \mathrm{MgCl}^{+}, \mathrm{Mg}_{2} \mathrm{CO}_{3}^{+2}$ and $\mathrm{MgCO}_{3}{ }^{\circ}$. The average concentration of these species, as compared to total soluble $\mathrm{Mg}$ in soil extract were : 40,91, 20,69, 38,22, 0,064 and 0,105\%,respectively. Finaly, the program showed that minerals controlling $\mathrm{Mg}$ activity in saline soils were either Mg-bearing calcite or dolomite.

Key-Words : Status, magnesium, salt, ions species, minérals and Irak. 


\section{INTRODUCTION}

Dans les régions arides et semi-arides, le climat rend l'irrigation nécessaire à toute mise en culture, ce qui exige une maîtrise des ressources en eau et en sol. Salim et Tessier (1998) ont mentionné que les facteurs de la formation des sols salés peuvent être purement climatiques ou induits par l'homme, notamment par l'utilisation de l'eau saline dans l'irrigation. En Irak, les eaux d'irrigations peuvent présenter des teneurs élevées en chlorures, sulfates et en carbonates de magnésium (Laribi, 2002). L'utilisation de ces eaux pour l'irrigation des sols agricoles peut engendrer une dégradation des caractéristiques physiques du sol. A cet effet, Dontsova et Norton (1999) ont montré que la présence du magnésium à des taux très élevés dans les sols, peut occasionner une dégradation de la structure du sol et une augmentation de l'érosion du sol. Ce processus de dégradation lié aux teneurs élevées en sodium échangeable a lieu en même temps que l'accumulation du magnésium dans le complexe d'échange (Curtin et al., 1994 ).

Il existe cependant, dans la littérature, très peu de travaux ayant étudié l'état du magnésium dans les sols salés. Nous pouvons citer les travaux de Daoud et al. (1993) sur quelques sols d'Algérie. Ces auteurs, ont observé une augmentation de la concentration en magnésium soluble avec la salinité du sol. Le magnésium soluble a représenté environ $20 \%$ de la somme des cations de la solution de sol (Daoud et al., 1993).

En Irak, l'étude faite par El-Hasani (1984) constitue une des références importantes en matière d'étude des propriétés des sols salés. Cet auteur a montré que l'irrigation des sols avec les eaux des fleuves Euphrate et Tigres, engendre une augmentation de la teneur en sels des sols. Les proportions de sels de magnésium et de calcium étaient de 76 et $90 \%$ du total.

L'objectif du présent travail est d'approfondir nos connaissances sur l'état du magnésium dans les sols salés. L'étude a porté sur le magnésium soluble étant donné qu'il intervient directement dans les processus physico-chimiques qui se déroulent dans la solution du sol.

\section{MATERIEL ET METHODES}

L'étude a été réalisée sur 86 échantillons de sols provenant des sols salés du centre et du sud de I'Irak (El-Falahi, 2000). La zone d'étude est caractérisée par l'abondance de sédiments apportés par les fleuves Euphrate et Tigres à la plaine alluviale mésopotamienne; en général, il s'agit de matériel calcaire avec un contenu en carbonate de calcium entre 15 et $35 \%$ dans les premiers centimètres de la couche superficielle (Laribi, 2005). Les conditions climatiques se caractérisent par une précipitation moyenne qui varie entre 10 et 18 centimètres par an. La température minimale peut descendre à $0{ }^{\circ} \mathrm{C}$ en Janvier. Les mois les plus chauds concernent la période de mai à fin septembre où on note une température très élevée en juillet, de l'ordre de $48{ }^{\circ} \mathrm{C}$. Les fractions granulométriques argileuses, limoneuses et sableuses ont été déterminées selon la méthode internationale à la pipette de Robinson. Les cations, les anions, la Capacité d'Echange de Cations (CEC) et le $\mathrm{pH}$ de sol ont été déterminés selon les méthodes décrites dans Richards (1954). Les teneurs en carbonate de calcium ont été déterminées par la méthode de Jackson (1976) et les carbonates de calcium actif selon la méthode de Galet (1972). Le calcul des différents sels dominants dans les sols et leurs pourcentages par rapport aux sels totaux a été réalisé selon la méthode de combinaison hypothétique préconisée par Zakharina (1963). Le principe de cette méthode repose sur le produit de solubilité des sels dans la solution du sol. Par ailleurs, en vue de connaître les différentes espèces ioniques et les différents minéraux contrôlant l'activité du magnésium dans les extraits de sols, le programme informatique Visual Minteq proposé par Gustafsson (2003) a été utilisé. La relation entre le magnésium soluble, les espèces ioniques et les propriétés des sols a fait l'objet de tests statistiques (coefficient de corrélations) au seuil de probabilité de $1 \%$ (Millar et Miller, 1993). 


\section{RESULTATS}

\section{CONCENTRATION DES DIFFERENTES FOR- MES DE MAGNESIUM DANS LES SOLS SALES}

Les données du Tableau 1 révèlent que les concentrations du magnésium soluble dans la solution de sol ont varié entre 155,4 et $686,6 \mathrm{meq} / \mathrm{l}$, soit en moyenne $378,5 \mathrm{meq} / \mathrm{l}$. Par ailleurs, le rapport $\mathrm{Mg} / \mathrm{Ca}$ dans la solution de sol a varié entre 1,64 et 7,58 , soit en moyenne 7,6. En revanche, les rapports $\mathrm{Mg} / \mathrm{Na}$ et $\mathrm{Mg} / \mathrm{K}$ dans la solution de sol ont varié entre 0,18 et 0,65 avec une moyenne de $0,4(\mathrm{Mg} / \mathrm{Na})$ et entre 19,01 et 271,38 , soit en moyenne $113,9(\mathrm{Mg} / \mathrm{K})$ respectivement. Dans le même sens, le taux du magnésium soluble par rapport à la somme des cations dans la solution du sol a varié entre 12,84 et $34,63 \%$, soit en moyenne $26,2 \%$.

Le rapport MgAR $(=\mathrm{Mg} /(\mathrm{Mg}+\mathrm{CA}) \times 100)$ a varié entre $62 \%$ et $94 \%$ soit en moyenne $84,6 \%$ pour tous les échantillons de sols (Tableau 1).

\section{RELATION DU MAGNESIUM SOLUBLE AVEC LES PROPRIETES DU SOL}

Les calculs statistiques relatifs aux corrélations simples montrent que la variation du magnésium en fonction de la CEC est significative $(r=$ $\left.0,72^{* \star}\right)$. La même tendance a été observée dans le cas de la réaction du sol $(\mathrm{pH})$. Le coefficient de corrélation entre ces deux variables est $(r=$ $0,73^{* *}$ ). Les relations statistiques ont été aussi significatives entre le magnésium soluble, le clore $\left(r=0,74^{* *}\right)$, le calcium $\left(r=0,5^{* *}\right)$ et le sodium $\left(r=0,54^{* *}\right)$.

Le traitement statistique des données a fait apparaître une relation significative, négative entre le magnésium soluble et la capacité d'échange cationique $\left(r=-0,46^{\star *}\right)$. En contrepartie, la relation était non significative avec la matière organique $(r=-0,19)$. Par ailleurs, les relations entre le magnésium soluble et les teneurs des autres variables ou caractéristiques des sols, notamment l'argile $(r=-0,03)$, limon $(r=0,03)$, sable $(r=$ $0,01)$, carbonates $(r=-0,06)$, calcaire total $(r=$ $0,13)$, calcaire actif $(r=0,10)$, potassium $(r=0,15)$ et sulfates $(r=0,03)$ ne sont pas significatives.

\section{SELS DE MAGNESIUM DOMINANTS DANS LES SOLS SALES}

Le tableau 2 expose les différents types de sels dominants dans les sols du centre et du sud de I'Irak. Les résultats obtenus, montrent que le sel le plus dominant dans les sols est le $\mathrm{NaCl}$. Les taux ont varié entre 38,52 et $83,12 \%$, soit en moyenne $57 \%$. La plus faible valeur a été observée dans les sols de Ouihda. La plus grande valeur a été signalée dans les sols de Shat El-Arab. De même, les résultats obtenus après combinaison hypothétique (Tableau 2) montrent que la majorité du magnésium est sous forme de $\mathrm{MgCl}_{2}$ et $\mathrm{MgSO}_{4}$, avec des taux de $\mathrm{MgCl}_{2}$ pour tous les sols variant entre 0,5 et $29,8 \%$, soit en moyenne $11,8 \%$. Les plus faibles valeurs sont observées dans les sols de Dhikar El- Rafaai et les plus élevées dans les sols de Sdinaouia. Concernant le $\mathrm{MgSO}_{4}$, sa valeur a varié pour tous les sols entre 2,28 et $27,66 \%$, soit en moyenne $13,8 \%$. Les sols de Dikhar El-Karmachia ont présenté les valeurs les plus élevées, par contre les plus faibles valeurs ont été observées dans les sols de Maissan. II ressort du tableau 2 que la valeur moyenne de sels de magnésium $\left(\mathrm{MgSO}_{4}+\mathrm{MgCl}_{2}+\mathrm{Mg}\left(\mathrm{HCO}_{3}\right)_{2}\right)$ dans les sols est de l'ordre de $25,6 \%$.

\section{FORMES IONIQUES DU MAGNESIUM}

L'utilisation du Visual Minteq a permis d'identifier la présence quatre formes de magnésium : $\mathrm{Mg}^{+}$, $\mathrm{MgSO}_{4}{ }^{0}, \mathrm{MgCl}^{+}, \mathrm{Mg}_{2} \mathrm{CO}_{3}{ }^{+2}$ et $\mathrm{MgCO}_{3}{ }^{\circ}$ (Figure 1).

La forme ionique $\mathrm{Mg}^{+}$a présenté une valeur qui a varié entre 30,61 et $55,95 \%$, soit en moyenne $40,91 \%$ du magnésium total soluble dans les extraits de sol. Quant à la forme $\mathrm{MgCl}^{+}$, elle a présenté des valeurs qui ont varié entre 15,45 et $56,23 \%$, soit en moyenne $38,22 \%$ du magnésium total soluble. L'espèce ionique $\mathrm{MgSO}_{4}{ }^{0}$ présente des valeurs qui ont varié entre 5,08 et $36,14 \%$, soit en moyenne $20,69 \%$ du magnésium total soluble. Concernant les autres espèces ioniques $\left(\mathrm{Mg}_{2} \mathrm{CO}_{3}{ }^{2+}\right.$ et $\left.\mathrm{MgCO}_{3}{ }^{0}\right)$, leurs taux sont relativement faibles, soit en moyenne $0,06 \%$ et $0,10 \%$ respectivement.

Des corrélations significatives ont été obtenues entre les différentes espèces ioniques et les propriétés physico-chimiques (Tableau 3 ). 


\section{MINERAUX MAGNESIENS DANS LES SOLS}

Le tableau 4 illustre les résultats du programme Visual Minteq relatifs aux différents minéraux magnésiens qui dominent dans les échantillons étudiés.Parmi les minéraux identifiés, il est distingué la présence de quatre minéraux essentiels : dolomite, epsomite, nesquehonite et $\mathrm{MgCO}_{3}, 5 \mathrm{H}_{2} \mathrm{O}$.

La comparaison du produit ionique IAP et du produit de solubilité KT (Tableau 4), nous a permis de relever :
- une sous-saturation vis-à-vis de la nesquehonite, l'epsomite et le $\mathrm{MgCO}_{3}, 5 \mathrm{H}_{2} \mathrm{O}$; l'indice de saturation est négatif ;

- une sous-saturation vis-à-vis de la dolomite pour les échantillons provenant des sites suivants : El-Djila, Maissan, Dikhar el Batha, Shat El-Arab, Dikhar El-Sdinaouia et Dikhar El-Rafaai ; l'indice de saturation est négatif ;

- une sur-saturation vis-à-vis de la dolomite pour le reste des sites à savoir Ouihda, El-Msaib, ElDiouania, Dikhar El-karmachia, Dikhar El-Karaf et Dikhar El-Shatra ; I'indice de saturation est positif.

Tableau 1 : Formes du magnésium dans les extraits de sols salés dans les différents sites d'étude Forms of Mg in soil extracts from the different study sites.

\begin{tabular}{|c|c|c|c|c|c|c|c|}
\hline Site d' étude & Mg meq/l & $\frac{\mathrm{Mg}^{+2}}{\mathrm{Ca}^{+2}}$ & $\frac{\mathrm{Mg}^{+2}}{\mathrm{~K}^{+}}$ & $\frac{\mathrm{Mg}^{+2}}{\mathrm{Na}^{+}}$ & MgAR & $\operatorname{MgAR}^{*}$ & $\operatorname{MgAR}{ }^{* *}$ \\
\hline Ouihda & 298,20 & 1,64 & 175,41 & 0,65 & 62,00 & 33,90 & 0,33 \\
\hline El-Msaib & 298,20 & 8,77 & 67,46 & 0,18 & 89,90 & 21,22 & 0,15 \\
\hline El-Djila & 370,74 & 5,24 & 106,23 & 0,25 & 84,00 & 19,09 & 0,19 \\
\hline El-Diouania & 371,00 & 13,74 & 58,51 & 0,33 & 93,00 & 23,35 & 0,24 \\
\hline Maissan & 686,60 & 4,52 & 271,38 & 0,43 & 82,00 & 29,75 & 0,28 \\
\hline Dikhar El-Batha & 582,00 & 4,39 & 53,98 & 0,34 & 81,00 & 25,30 & 0,24 \\
\hline Shat El-Arab & 425,40 & 7,13 & 19,01 & 0,18 & 87,00 & 12,84 & 0,15 \\
\hline Dikhar El-Karmachia & 155,40 & 4,89 & 114,26 & 0,57 & 83,00 & 34,63 & 0,34 \\
\hline Dikhar El-Sdinaouia & 562,00 & 4,03 & 136,73 & 0,65 & 80,00 & 32,61 & 0,36 \\
\hline Dikhar El-Karaf & 319,60 & 7,13 & 110,97 & 0,38 & 87,00 & 30,95 & 0,25 \\
\hline Dikhar El-Shatra & 281,40 & 17,58 & 87,39 & 0,33 & 94,0 & 25,18 & 0,24 \\
\hline Dikhar El-Rafaai & 299,60 & 12,7 & 165,52 & 0,37 & 93,0 & 25,89 & 0,26 \\
\hline Moyenne & 387,5 & 7,6 & 113,9 & 0,4 & 84,6 & 26,2 & 0,20 \\
\hline
\end{tabular}

$\mathrm{MgAR}=\mathrm{Mg}^{+2} / \mathrm{Mg}^{+2}+\mathrm{Ca}^{+2}, 100, \mathrm{MgAR}^{*}=\mathrm{Mg}^{+2} / \mathrm{Mg}^{+2}+\mathrm{Ca}^{+2}+\mathrm{Na}^{+}+\mathrm{K}^{+}, 100, \mathrm{MgAR}^{* *}=\mathrm{Mg}^{+2} / \mathrm{Cl}^{+}+\mathrm{SO}_{4}^{-2}+\mathrm{HCO}_{3}$

Tableau 2 : Sels dominants (\%) dans les extraits de sols.

Most important salts (\%) in soil extract.

\begin{tabular}{lcccccccc}
\hline Sites d'études & $\mathrm{NaCl}$ & $\mathrm{Na}_{2} \mathrm{SO}_{4}$ & $\mathrm{MgCl}_{2}$ & $\mathrm{MgSO}_{4}$ & $\left.\mathrm{Mg} \mathrm{HCO}_{3}\right)_{2}$ & $\mathrm{CaCl}_{2}$ & $\mathrm{CaSO}_{4}$ & $\mathrm{Ca}\left(\mathrm{HCO}_{3}\right)_{2}$ \\
\hline Ouihda & 38,52 & 1,79 & 18,36 & 14,20 & 0,086 & 8,55 & 4,74 & 2,20 \\
El-Msaib & 58,56 & 21,33 & 4,97 & 9,47 & 0 & 0 & 2,41 & 0,24 \\
El-Djila & 65,04 & 11,88 & 10,05 & 9,079 & 0 & 0 & 3,761 & 0,167 \\
El-Diouania & 58,42 & 15,72 & 9,24 & 14,25 & 0 & 0 & 1,77 & 0,52 \\
Maissan & 62,33 & 0 & 27,52 & 2,28 & 0 & 3,30 & 4,33 & 0,221 \\
Dikhar El-Batha & 66,33 & 2,49 & 17,58 & 7,88 & 0 & 0,87 & 4,7 & 0,1 \\
Shat El-Aarab & 83,12 & 0 & 7,20 & 6,04 & 0 & 0,20 & 2,28 & 0,12 \\
Di-Khar El-Karmachia & 51,79 & 5,87 & 7,04 & 27,66 & 0 & 0,05 & 5,22 & 2,87 \\
Dikhar El-Sdinaouia & 46,63 & 0 & 29,80 & 2,49 & 0 & 7,68 & 10 & 0,27 \\
Dikhar El-Karaf & 48,67 & 15,73 & 8,40 & 22,62 & 0,23 & 0 & 3,61 & 0,71 \\
Dikhar El-Shatra & 50,94 & 21,58 & 0,71 & 24,47 & 0,073 & 0 & 1,22 & 0,96 \\
Dikhar El-Rafaai & 51,25 & 20,68 & 0,50 & 25,44 & 0 & 0 & 1,67 & 0,44 \\
Moyenne & 57 & 9,75 & 11,8 & 13,82 & 0,0324 & 1,72 & 3,8 & 0,54 \\
\hline
\end{tabular}




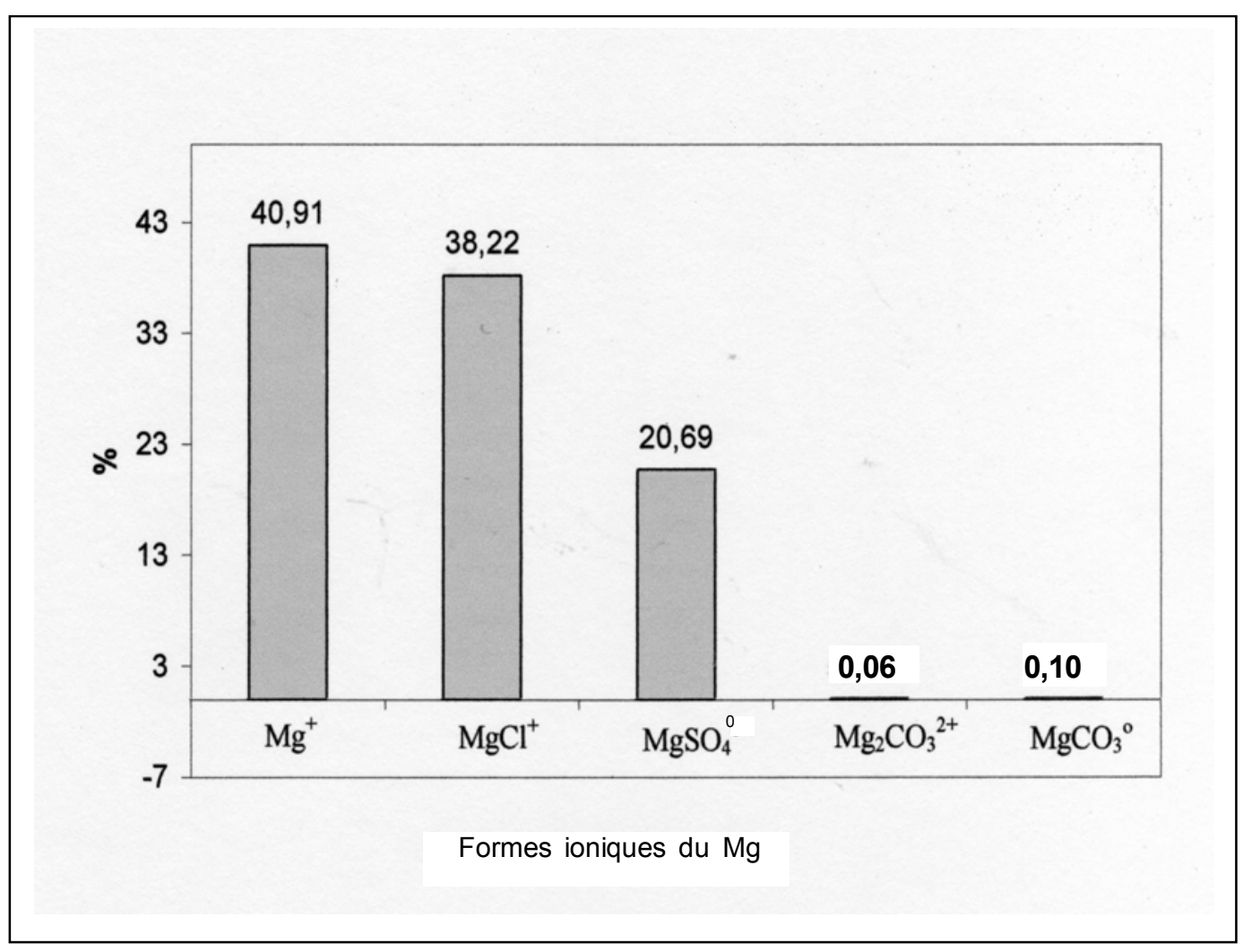

Figure 1 : Différentes formes ioniques du magnésium.

Different magnesium ions species.

Tableau 3 : Coefficient de corrélation $(r)$ entre espèces ioniques et propriétés des sols.

Correlation coefficient ( $r$ ) between ions species and soil properties.

\begin{tabular}{lllll}
\hline (r) & $\mathrm{Mg}^{+}$ & $\mathrm{MgCl}^{+}$ & $\mathrm{MgSO}_{4}{ }^{0}$ & $\mathrm{Mg}_{2} \mathrm{CO}_{3}{ }^{2+}$ \\
\hline $\mathrm{pH}$ & $\mathrm{r}=0.54^{* *}$ & $\mathrm{r}=-0.95^{\star *}$ & $\mathrm{r}=0.87^{* *}$ & $\mathrm{r}=0.69^{* *}$ \\
$\mathrm{CEC}$ & $\mathrm{r}=-0.88^{* *}$ & $\mathrm{r}=0.89^{* *}$ & $\mathrm{r}=-0.58^{* *}$ & $\mathrm{r}=-0.76^{* *}$ \\
\hline
\end{tabular}


Tableau 4 : Différents minéraux magnésiens dans les échantillons de sol étudiés. (Output Visual Minteq). Diferent magnesian minerals in studied soil samples.

\begin{tabular}{|c|c|c|c|c|c|c|c|c|c|}
\hline \multirow{5}{*}{$\begin{array}{l}\text { Site } \\
\text { Ouinda }\end{array}$} & \multirow{2}{*}{$\begin{array}{l}\text { Minéraux } \\
\text { Dolomite }\end{array}$} & \multirow{2}{*}{$\begin{array}{l}\log \text { IAP } \\
-13,089\end{array}$} & \multirow{2}{*}{$\begin{array}{c}\begin{array}{c}\text { Produit de } \\
\text { solubilité } \\
\text { (KT) }\end{array} \\
3,451\end{array}$} & \multicolumn{6}{|c|}{ Composantes minérales et stoechiométrique } \\
\hline & & & & 1 & $\mathrm{Ca}^{+2}$ & 1 & $\mathrm{Mg}^{+2}$ & 2 & $\mathrm{CO}_{3}^{-2}$ \\
\hline & Epsomite & $-4,140$ & $-2,013$ & 1 & $\mathrm{Mg}^{+2}$ & 1 & $\mathrm{SO}_{4}^{-2}$ & 7 & $\mathrm{H}_{2} \mathrm{O}$ \\
\hline & Nesquehonite & $-6,476$ & 1,806 & 1 & $\mathrm{Mg}^{+2}$ & 1 & $\mathrm{CO}_{3}^{-2}$ & 3 & $\mathrm{H}_{2} \mathrm{O}$ \\
\hline & $\mathrm{MgCO}_{3}: 5 \mathrm{H}_{2} \mathrm{O}$ & $-6,499$ & $-1,959$ & 1 & $\mathrm{Mg}^{+2}$ & 1 & $\mathrm{CO}_{3}^{-2}$ & 5 & $\mathrm{H}_{2} \mathrm{O}$ \\
\hline \multirow{4}{*}{ El-Msaib } & Dolomite & $-14,318$ & 2,222 & 1 & $\mathrm{Ca}^{+2}$ & 1 & $\mathrm{Mg}^{+2}$ & 2 & $\mathrm{CO}_{3}^{-2}$ \\
\hline & Epsomite & $-3,540$ & $-1,414$ & 1 & $\mathrm{Mg}^{+2}$ & 1 & $\mathrm{SO}_{4}^{-2}$ & 7 & $\mathrm{H}_{2} \mathrm{O}$ \\
\hline & Nesquehonite & $-6,741$ & $-2,071$ & 1 & $\mathrm{Mg}^{+2}$ & 1 & $\mathrm{CO}_{3}^{-2}$ & 3 & $\mathrm{H}_{2} \mathrm{O}$ \\
\hline & $\mathrm{MgCO}_{3}: 5 \mathrm{H}_{2} \mathrm{O}$ & $-6,787$ & $-2,247$ & 1 & $\mathrm{Mg}^{+2}$ & 1 & $\mathrm{CO}_{3}^{-2}$ & 5 & $\mathrm{H}_{2} \mathrm{O}$ \\
\hline \multirow{4}{*}{ El-Djila } & Dolomite & $-39,350$ & $-22,810$ & 1 & $\mathrm{Ca}^{+2}$ & 1 & $\mathrm{Mg}^{+2}$ & 2 & $\mathrm{CO}_{3}{ }^{-2}$ \\
\hline & Epsomite & $-3,683$ & $-1,556$ & 1 & $\mathrm{Mg}^{+2}$ & 1 & $\mathrm{SO}_{4}^{-2}$ & 7 & $\mathrm{H}_{2} \mathrm{O}$ \\
\hline & Nesquehonite & $-19,386$ & $-14,716$ & 1 & $\mathrm{Mg}^{+2}$ & 1 & $\mathrm{CO}_{3}^{-2}$ & 3 & $\mathrm{H}_{2} \mathrm{O}$ \\
\hline & $\mathrm{MgCO}_{3}: 5 \mathrm{H}_{2} \mathrm{O}$ & $-19,431$ & $-14,891$ & 1 & $\mathrm{Mg}^{+2}$ & 1 & $\mathrm{CO}_{3}^{-2}$ & 5 & $\mathrm{H}_{2} \mathrm{O}$ \\
\hline \multirow{4}{*}{ El-Diouania } & Dolomite & $-14,252$ & 2,288 & 1 & $\mathrm{Ca}^{+2}$ & 1 & $\mathrm{Mg}^{+2}$ & 2 & $\mathrm{CO}_{3}^{-2}$ \\
\hline & Epsomite & $-3,640$ & $-1,514$ & 1 & $\mathrm{Mg}^{+2}$ & 1 & $\mathrm{SO}_{4}^{-2}$ & 7 & $\mathrm{H}_{2} \mathrm{O}$ \\
\hline & Nesquehonite & $-6,613$ & $-1,943$ & 1 & $\mathrm{Mg}^{+2}$ & 1 & $\mathrm{CO}_{3}^{-2}$ & 3 & $\mathrm{H}_{2} \mathrm{O}$ \\
\hline & $\mathrm{MgCO}_{3}: 5 \mathrm{H}_{2} \mathrm{O}$ & $-6,653$ & $-2,113$ & 1 & $\mathrm{Mg}^{+2}$ & 1 & $\mathrm{CO}_{3}^{-2}$ & 5 & $\mathrm{H}_{2} \mathrm{O}$ \\
\hline \multirow{4}{*}{ Maissan } & Dolomite & $-39,030$ & $-22,490$ & 1 & $\mathrm{Ca}^{+2}$ & 1 & $\mathrm{Mg}^{+2}$ & 2 & $\mathrm{CO}_{3}^{-2}$ \\
\hline & Epsomite & $-3,977$ & $-1,850$ & 1 & $\mathrm{Mg}^{+2}$ & 1 & $\mathrm{SO}_{4}^{-2}$ & 7 & $\mathrm{H}_{2} \mathrm{O}$ \\
\hline & Nesquehonite & $-19,279$ & $-14,609$ & 1 & $\mathrm{Mg}^{+2}$ & 1 & $\mathrm{CO}_{3}^{-2}$ & 3 & $\mathrm{H}_{2} \mathrm{O}$ \\
\hline & $\mathrm{MgCO}_{3}: 5 \mathrm{H}_{2} \mathrm{O}$ & $-19,325$ & $-14,785$ & 1 & $\mathrm{Mg}^{+2}$ & 1 & $\mathrm{CO}_{3}^{-2}$ & 5 & $\mathrm{H}_{2} \mathrm{O}$ \\
\hline \multirow{4}{*}{ Dikhar El-Batha } & Dolomite & $-39,102$ & $-22,562$ & 1 & $\mathrm{Ca}^{+2}$ & 1 & $\mathrm{Mg}^{+2}$ & 2 & $\mathrm{CO}_{3}^{-2}$ \\
\hline & Epsomite & $-3,795$ & $-1,668$ & 1 & $\mathrm{Mg}^{+2}$ & 1 & $\mathrm{SO}_{4}^{-2}$ & 7 & $\mathrm{H}_{2} \mathrm{O}$ \\
\hline & Nesquehonite & $-19,316$ & $-14,646$ & 1 & $\mathrm{Mg}^{+2}$ & 1 & $\mathrm{CO}_{3}^{-2}$ & 3 & $\mathrm{H}_{2} \mathrm{O}$ \\
\hline & $\mathrm{MgCO}_{3}: 5 \mathrm{H}_{2} \mathrm{O}$ & $-19,361$ & $-14,821$ & 1 & $\mathrm{Mg}^{+2}$ & 1 & $\mathrm{CO}_{3}^{-2}$ & 5 & $\mathrm{H}_{2} \mathrm{O}$ \\
\hline \multirow{4}{*}{ Shat El-Aarab } & Dolomite & $-39,506$ & $-22,966$ & 1 & $\mathrm{Ca}^{+2}$ & 1 & $\mathrm{Mg}^{+2}$ & 2 & $\mathrm{CO}_{3}{ }^{-2}$ \\
\hline & Epsomite & $-3,990$ & $-1,863$ & 1 & $\mathrm{Mg}^{+2}$ & 1 & $\mathrm{SO}_{4}^{-2}$ & 7 & $\mathrm{H}_{2} \mathrm{O}$ \\
\hline & Nesquehonite & $-19,419$ & $-14,749$ & 1 & $\mathrm{Mg}^{+2}$ & 1 & $\mathrm{CO}_{3}^{-2}$ & 3 & $\mathrm{H}_{2} \mathrm{O}$ \\
\hline & $\mathrm{MgCO}_{3}: 5 \mathrm{H}_{2} \mathrm{O}$ & $-19,464$ & $-14,924$ & 1 & $\mathrm{Mg}^{+2}$ & 1 & $\mathrm{CO}_{3}^{-2}$ & 5 & $\mathrm{H}_{2} \mathrm{O}$ \\
\hline \multirow{4}{*}{ Dikhar El-Karmachia } & Dolomite & $-13,172$ & 3,368 & 1 & $\mathrm{Ca}^{+2}$ & 1 & $\mathrm{Mg}^{+2}$ & 2 & $\mathrm{CO}_{3}{ }^{-2}$ \\
\hline & Epsomite & $-3,918$ & $-1,791$ & 1 & $\mathrm{Mg}^{+2}$ & 1 & $\mathrm{SO}_{4}^{-2}$ & 7 & $\mathrm{H}_{2} \mathrm{O}$ \\
\hline & Nesquehonite & $-6,239$ & $-1,569$ & 1 & $\mathrm{Mg}^{+2}$ & 1 & $\mathrm{CO}_{3}^{-2}$ & 3 & $\mathrm{H}_{2} \mathrm{O}$ \\
\hline & $\mathrm{MgCO}_{3}: 5 \mathrm{H}_{2} \mathrm{O}$ & $-6,250$ & $-1,710$ & 1 & $\mathrm{Mg}^{+2}$ & 1 & $\mathrm{CO}_{3}^{-2}$ & 5 & $\mathrm{H}_{2} \mathrm{O}$ \\
\hline \multirow{4}{*}{ Dikar El-Sdinaouia } & Dolomite & $-39,043$ & $-22,503$ & 1 & $\mathrm{Ca}^{+2}$ & 1 & $\mathrm{Mg}^{+2}$ & 2 & $\mathrm{CO}_{3}^{-2}$ \\
\hline & Epsomite & $-3,833$ & $-1,707$ & 1 & $\mathrm{Mg}^{+2}$ & 1 & $\mathrm{SO}_{4}^{-2}$ & 7 & $\mathrm{H}_{2} \mathrm{O}$ \\
\hline & Nesquehonite & $-19,291$ & $-14,621$ & 1 & $\mathrm{Mg}^{+2}$ & 1 & $\mathrm{CO}_{3}^{-2}$ & 3 & $\mathrm{H}_{2} \mathrm{O}$ \\
\hline & $\mathrm{MgCO}_{3}: 5 \mathrm{H}_{2} \mathrm{O}$ & $-19,332$ & $-14,792$ & 1 & $\mathrm{Mg}^{+2}$ & 1 & $\mathrm{CO}_{3}^{-2}$ & 5 & $\mathrm{H}_{2} \mathrm{O}$ \\
\hline \multirow{4}{*}{ Dikhar El-Karaf } & Dolomite & $-14,022$ & 2,518 & 1 & $\mathrm{Ca}^{+2}$ & 1 & $\mathrm{Mg}^{+2}$ & 2 & $\mathrm{CO}_{3}^{-2}$ \\
\hline & Epsomite & $-3,529$ & $-1,402$ & 1 & $\mathrm{Mg}^{+2}$ & 1 & $\mathrm{SO}_{4}^{-2}$ & 7 & $\mathrm{H}_{2} \mathrm{O}$ \\
\hline & Nesquehonite & $-6,615$ & $-1,945$ & 1 & $\mathrm{Mg}^{+2}$ & 1 & $\mathrm{CO}_{3}^{-2}$ & 3 & $\mathrm{H}_{2} \mathrm{O}$ \\
\hline & $\mathrm{MgCO}_{3}: 5 \mathrm{H}_{2} \mathrm{O}$ & $-6,646$ & $-2,106$ & 1 & $\mathrm{Mg}^{+2}$ & 1 & $\mathrm{CO}_{3}^{-2}$ & 5 & $\mathrm{H}_{2} \mathrm{O}$ \\
\hline \multirow{4}{*}{ Dikhar El-Shatra } & Dolomite & $-13,821$ & 2,719 & 1 & $\mathrm{Ca}^{+2}$ & 1 & $\mathrm{Mg}^{+2}$ & 2 & $\mathrm{CO}_{3}^{-2}$ \\
\hline & Epsomite & $-3,561$ & $-1,434$ & 1 & $\mathrm{Mg}^{+2}$ & 1 & $\mathrm{SO}_{4}^{-2}$ & 7 & $\mathrm{H}_{2} \mathrm{O}$ \\
\hline & Nesquehonite & $-6,313$ & $-1,643$ & 1 & $\mathrm{Mg}^{+2}$ & 1 & $\mathrm{CO}_{3}^{-2}$ & 3 & $\mathrm{H}_{2} \mathrm{O}$ \\
\hline & $\mathrm{MgCO}_{3}: 5 \mathrm{H}_{2} \mathrm{O}$ & $-6,342$ & $-1,802$ & 1 & $\mathrm{Mg}^{+2}$ & 1 & $\mathrm{CO}_{3}^{-2}$ & 5 & $\mathrm{H}_{2} \mathrm{O}$ \\
\hline \multirow{4}{*}{ Dikhar El-Rafaai } & Dolomite & $-39,689$ & $-23,149$ & 1 & $\mathrm{Ca}^{+2}$ & 1 & $\mathrm{Mg}^{+2}$ & 2 & $\mathrm{CO}_{3}{ }^{-2}$ \\
\hline & Epsomite & $-3,516$ & $-1,389$ & 1 & $\mathrm{Mg}^{+2}$ & 1 & $\mathrm{SO}_{4}^{-2}$ & 7 & $\mathrm{H}_{2} \mathrm{O}$ \\
\hline & Nesquehonite & $-19,299$ & $-14,629$ & 1 & $\mathrm{Mg}^{+2}$ & 1 & $\mathrm{CO}_{3}^{-2}$ & 3 & $\mathrm{H}_{2} \mathrm{O}$ \\
\hline & $\mathrm{MgCO}_{3}: 5 \mathrm{H}_{2} \mathrm{O}$ & $-19,327$ & $-14,787$ & 1 & $\mathrm{Mg}^{+2}$ & 1 & $\mathrm{CO}_{3}^{-2}$ & 5 & $\mathrm{H}_{2} \mathrm{O}$ \\
\hline
\end{tabular}

Indice de saturation $=$ Log IAP-Log KT ; IAP : Produit d'activité ionique ; KT : Produit de solubilité. 


\section{DISCUSSION}

L'étude de différentes formes du magnésium dans les sols salés a montré que le taux de magnésium soluble par rapport à la somme des cations dans la solution du sol représente en moyenne $26,2 \%$. Ce résultat est semblable a celui rapporté par Boulaine (1957), Peters et Ghoul (1972) et Daoud (1983) sur les niveaux de magnésium enregistrés dans quelques sols d'Algérie. D'autre part, l'étude a révélé un rapport MgAR en moyenne de l'ordre de 84,6\%. Le résultat trouvé dans cette étude est supérieur à la valeur seuil mentionnée par Kovda (1973), qui est de l'ordre de $50 \%$. Ceci présente pour les cultures, des risques de toxicité liés d'une part, aux niveaux très élevés de magnésium et, d'autre part, à la salinité. Les résultats sont comparables à ceux obtenus par Fink et Chleiff (1975) qui ont signalé des niveaux très élevés de magnésium dans quelques sols d'Italie. Ces auteurs ont observé une baisse des rendements de blé pour des concentrations en magnésium soluble supérieures à $1000 \mathrm{ppm}$. Cette baisse de rendement, a été prépondérante dans les sols contenant des sels de chlorures de magnésium lorsqu'on les compare aux sols caractérisés par la présence de sels de sulfates de magnésium (Fink et Chleiff, 1975).

Le magnésium soluble a présenté une corrélation significative avec la salinité. Cette relation peut s'expliquer par le fait que l'accumulation du magnésium soluble dans les sols salés se produit en même temps que celle des sels dans les zones arides et semi-arides. Daoud et al. (1993) et Ubald et al. (1994) ont également observé la même corrélation entre ces deux variables.

L'autre paramètre qui influence la mobilité du magnésium dans le sol, est la réaction du sol $(\mathrm{pH})$. Le traitement statistique a révélé une corrélation significative négative. L'augmentation du $\mathrm{pH}$ du sol entraîne une diminution de la concentration de magnésium soluble dans les sols calcaires. Cela est lié vraisemblablement à sa précipitation sous forme de carbonate de calcium (Miyazawa et al., 2001). C'est le cas de nos échantillons de sols qui présentent des taux de calcaire supérieurs à $25 \%$ (Laribi, 2002).

La matière organique présente une corrélation non significative mais négative avec le magnésium soluble. Selon Schnitzer et Skinner
(1967) et Sposito (1977), la matière organique forme avec le magnésium des composés qui entraînent sa fixation. Cependant, la non significativité de la relation serait liée à la faible variation du taux de matière organique des sols étudiés (Laribi, 2002).

La capacité d'échange cationique exerce aussi un effet important dans la rétention du magnésium. Le traitement statistique a révélé une corrélation significative entre ces deux variables. Au fur et à mesure qu'augmentent les sites d'adsorption du complexe argilo-humique, la concentration du magnésium adsorbé augmente et sa teneur dans la solution du sol diminue.

Parmi les minéraux magnésiens dominants identifiés, quatre sont essentiels : dolomite, epsomite, nesquehonite et $\mathrm{MgCO}_{3}, 5 \mathrm{H}_{2} \mathrm{O}$. Ces minéraux sont susceptibles de contrôler l'activité du magnésium.

Les résultats de l'étude des minéraux magnésiens concordent avec ceux rapportées par Deffeyes et al. (1964) et Bui et al. (1990) qui ont signalé la possibilité de précipitation du magnésium sous forme de dolomite dans le sol. Selon ces derniers auteurs, la précipitation de la dolomite se produit quand le rapport $\mathrm{Mg}^{+2}$ / $\mathrm{Mg}^{+2}+\mathrm{Ca}^{+2}$ est supérieur à 0,25 . Dans nos conditions d'étude, ce rapport à varié de 0,62 à 0,94 , soit une moyenne de 0,84 .

St-Arnaud et Herbillon (1973) et Berner (1975) ont signalé que les minéraux contenant du Mg (Mg-bearing Calcite) précipitent dans un système ou le rapport $\mathrm{Mg}^{+2} / \mathrm{Ca}^{+2}$ est supérieur à 1 . Nos données montrent des valeurs qui ont varié entre 1,64 et 17,58 soit en moyenne 7,6.

Toutefois, ces résultats doivent être pris avec réserve car, selon Bohn et al. (2001), la précipitation de la dolomite dépend de la réaction du sol et elle ne se manifeste qu'à des $\mathrm{pH}$ supérieurs à 9,5 ; ce qui n'est pas le cas des échantillons étudiés qui présentent un $\mathrm{pH}$ neutre (Laribi, 2002).

\section{CONCLUSION}

Cette étude a montré que la majeure partie des sols étudiés ont présenté des niveaux très élevés en magnésium soluble. Ce résultat doit être pris en compte lors de l'aménagement et la gestion de ces sols. 
Les résultats de la combinaison hypothétique ont montré que la majorité des sels de magnésium était sous forme de $\mathrm{MgCl}_{2}, \mathrm{MgSO}_{4}$. La quantité de $\mathrm{Mg}\left(\mathrm{HCO}_{3}\right)_{2}$ était négligeable.

L'application du logiciel Visual Minteq a mis en évidence les formes ioniques dans l'ordre de dominance suivant en fonction de leurs pourcentages: $\mathrm{Mg}^{+2}>\mathrm{MgCl}^{+}>\mathrm{MgSO}_{4}{ }^{0}$ $>\mathrm{MgCO}_{3}{ }^{0}>\mathrm{Mg}_{2} \mathrm{CO}_{3}{ }^{+2}$.

Le calcul des rapports du magnésium aux différents cations-spéciation a montré que les minéraux, qui peuvent contrôler l'activité du magnésium dans les sols, peuvent être soit des calcaires contenant du magnésium ( $\mathrm{Mg}$ bearing calcite) soit de la dolomite. Cependant, ces constats mériteraient d'être approfondis par des analyses minéralogiques et des observations aux rayons $X$, afin d'identifier la possibilité de précipitation de la dolomite dans les sols étudiés.

\section{REFERENCES}

Berner R. A. 1975.The role of magnesium in the growth of calcite and aragonite from sea water. Geochim. Cosmochim. Acta 39 : $489-504$

Bohn H. L., B. L. Mc. Neal and G. A. O cornner. 2001. Soil chemistry $3^{\text {rd }}$ ed. John Wiley and Sons. New York, 307 p.

Boulaine S. 1957. Les sols des plaines du Cheliff. Thèse Doctorat d'état. Université d'Alger $357 \mathrm{p}$.

Bui E. N., R. H. Leoppert and L. P. Wilding. 1990. Carbonates phases in calcareous soils of the western unated states. Soil Sc. Soc. Am. J. $54: 39-45$.

Curtin D., H. Steppuhn and F. Selles. 1994. Effects of magnesium on cation selectivity and structural stability of sodic soils. Soil. Sci. Soc. Am. J. 58 : 737 - 739 .

Daoud Y. 1983. Contribution à l'étude de la dynamique des sels dans un sol irrigué du périmètre du haut-Cheliff (Algérie). Thèse Doct. Ing. ENSA. Université de Rennes, $194 \mathrm{p}$.

Daoud Y., C. Cheverry et M. Robert. 1993. Rôle physico-chimique du magnésium dans les sols salés de la plaine du Cheliff (Algérie) Science du sol. 31 (4) : 281 - 293.

Dontsova K. and D. L. Norton. 1999. Effects of exchangeable $\mathrm{Ca}$ : $\mathrm{Mg}$ ratio on soil clay flocculation, infiltration and erosion. Proceeding of $10^{\text {th }}$ International Soil Conservation Organization Meeting held May 24 - 29, at Parue University and the USDA-ARS National Soil Erosion Research Laboratory : pp 580 - 585.

Deffeyes K. S., F. J. Lucia and P. K. Weyl. 1964 Dolomitization : observation on the Island of Bonaire, Netherlands Antilles.Science. $143: 678-679$.

El-Hassani A. A. 1984. Properties study of sabkha and shoura soils of Iraq. MSc thesis. College of Agriculture.Baghdad University, $95 \mathrm{p}$

El-Falahi A. A. 2000. Status and behavior of boron in saline soils of Iraq. PhD thesis. College of Agriculture. Baghdad University, 131 p.

Finck A. and U. Schleiff. 1975. Salt tolerance of crops under ecological conditions in southern Italy with special reference to high magnesium content in soil. Proceeding of the International Symposium on new developments in the field of salt affected soils. Cairo : pp 627 - 633.

Galet D. 1972. Dosage du calcaire actif. Méthodes d'analyses du laboratoire. Division technique. Solaigue Nimes. France : pp 37 - 38.

Gustafsson J. P. 2003. Visual Minteq : A computer program for the calculating of chemical equilibria. Dept of Land and Water Resources Engineering. Stockholm, Sweden.

Jackson M. L. 1976. Analisis químico de suelos. Ediciones Omega, S. A. Barcelona, 662 p.

Kovda V. 1973. Irrigation/Drainage and Salinity. International source book FAO/UNESCO, $123 \mathrm{p}$.

Laribi A. 2002. Status and Behavior of Magnesium in saline soils of Iraq. MSc thesis. College of Agriculture. Baghdad University, 107 p.

Laribi A. 2005. Estudio de comportamiento del magnesio frente al calcio en suelos aluviales. Trabajo de investigación. Centro de Investigaciones Sobre Desertificación. CIDE, Albal. Universidad de Valencia, $52 \mathrm{p}$.

Lindsay W. L. 1979. Chemical equilibrium in soil. John Wiley, N. Y., 449 p.

Millar J. C. And J. N. Miller. 1993. Estadistica para química analitica, Segunda edición. Addison-Wesley Iberoamerican, 211p. 
Miyazawa M., Pavan M. A., Ziglio C. A. and J. C. Franchini. 2001. Reduction of Exchan-geable Calcium and Magnesium in Soil with Increasing pH. Braz. arch. Biol. Technol. 4(2) : $149-153$

Peters J. et S. A. Ghoul. 1972. Etude agropédologique du périmètre irrigué de la mina. I. N. R. H. Alger, $54 \mathrm{p}$.

Richards L. A. 1954. Diagnosis and improvement of saline álcali soils. USDA Handbook. $\mathrm{N}^{\circ} 60,172 \mathrm{p}$.

Salim S. et D. Tessier. 1998. Evolution des propriétés physiques et physico-chimiques des sols salés de la basse vallée de l'Euphrate (Syrie). Etude et gestion des sols. 5 (4) : 277 - 288.

Schnitzer M. and S. I. M. Skinner. 1967. Organometallic interactions in soil. Stability constant of $\mathrm{Pb}^{+2}, \mathrm{Ni}^{+2}, \mathrm{Ca}^{+2}$, and $\mathrm{Mg}^{+2}$ fulvic acid complexes. Soil Sci. $103: 247$ - 252.
Sposito G., K. M. Hlaltz claw and C. S. Levesque. 1977. Calcium ion complexation by fulvic and extracts from sewage sludge-soil mixtures. Soil. Sci. Soc. Am. J. 42 : $600-606$.

St-Arnaud R. J and A. J. Herbillon. 1973. Occurrence and genesis of secondary magnesium bearing calcites in soils. Geoderma. $9: 279$ - 298.

Ubald G. G., J. J. Stephan and H. Sticher. 1994. Chemistry and speciation of soil water from serpentinic soil : importance of colloids in the teansport of $\mathrm{Cr}, \mathrm{Fe}, \mathrm{Mg}$ and Ni. Soil. Sci : 158 (5) : 314 - 322.

Zakharina G. V. 1963. Problem concerning the classification of natural waters and solutions. Sov. Soil. Sci. 4 : $351-362$ 